\title{
ANÁLISIS DE LA COMUNICACIÓN EN LA PROMOCIÓN DEL CAMINO DE SANTIAGO COMO PRODUCTO TURÍSTICO
}

\author{
Silvia Mondelo López ${ }^{1}$ \\ Magdalena Rodríguez Fernández ${ }^{2}$ \\ Universidad de A Coruña \\ silvia.mondelo@gmail.com \\ mmrodriguez@udc.es
}

Material autorizado para su primera publicación en la revista académica REDMARKA como artículo.

https://doi.org/10.17979/redma.2011.01.06.4720

\section{RESUMEN}

El Camino de Santiago ${ }^{3}$ se ha ido consolidando con el paso de los años en uno de los atractivos turísticos más importantes de Galicia. Prueba de ello es que gran parte de los visitantes de la Comunidad Autónoma han llegado directa o indirectamente a través de esta ruta de peregrinación milenaria.

Precisamente, uno de los aspectos clave a la hora de dar a conocer el Camino tanto a nivel nacional como internacional han sido las diversas campañas de comunicación y promoción desarrolladas al respecto. Es por ello que el objetivo central de este artículo se fundamenta en analizar la promoción llevada a cabo

\footnotetext{
${ }^{1}$ Diplomada en Turismo por la Universidad de A Coruña; Master en Dirección y Planificación del Turismo por la Universidad de A Coruña.

${ }^{2}$ Doctora en Ciencias Económicas y Empresariales por la Universidad de A Coruña; Postgrado en Economía y Calidad en el Sector turístico por la Universidad de A Coruña; Licenciada en Ciencias Económicas y Empresariales por la Universidad de Santiago de Compostela; Docente en la Escuela Universitaria de Turismo y en el Máster Oficial en Dirección y Planificación del Turismo de la Universidad de A Coruña.

${ }^{3}$ Constituye todo un conjunto de rutas de peregrinación que tienen como meta el sepulcro del apóstol Santiago el Mayor en Compostela. Considerado como el primer Itinerario Cultural Europeo y declarado Patrimonio de la Humanidad en 1993. Entre las diversas rutas que los conforman el Camino Francés, se ha convertido en el camino por definición al ser el más concurrido y conocido.
} 
en torno al Camino de Santiago como producto turístico ${ }^{4}$, tomando como especial referencia las acciones desarrolladas durante el pasado Año Santo ${ }^{5}$ o Xacobeo $^{6} 2010$.

Para la realización de esta investigación, la metodología utilizada se ha basado en el empleo de fuentes primarias. Las cuales se han fundamentado en la elaboración y realización de entrevistas en profundidad a un grupo de personas vinculadas, de una manera $u$ otra, con el Camino de Santiago y su comunicación.

Palabras clave: Comunicación, Promoción, Producto turístico, Camino de Santiago.

\section{ABSTRACT}

COMMUNICATION ANALYSIS IN THE PROMOTION OF WAY OF ST. JAMES AS TOURISM PRODUCT

The Way of St. James has been consolidating over the years into one of the most important tourist attractions of Galicia. Proof of this is that many of the visitors of the Autonomous Region have come directly or indirectly through this ancient pilgrimage route.

Indeed, one of the key aspects when to share the road both domestically and internationally have been the various communication and promotion campaigns developed in this regard. That is why the aim of this paper is based on analyzing the promotion that took place about the Way of St. James as a

\footnotetext{
${ }^{4}$ Producto turístico: conjunto de elementos tangibles e intangibles, naturales y artificiales, que actúan de forma interrelacionada para cubrir las necesidades de los turistas (Aguirre, 2000). En este sentido Marchena (1993) define el Camino de Santiago como producto turístico ya que puede considerarse como una de las primeras rutas turísticas de la historia, contando con todos los elementos necesarios para ser observada como tal strictu senso: oferta de alojamiento (albergue de peregrinos), oferta complementaria (posadas, mesones,...), recursos turísticos (monumentales, históricos, naturales,...) y hasta incluso su propia guía turística, el Liber Peregrinationis.

${ }^{5}$ Cuando la festividad del apóstol Santiago (25 de julio) coincide en domingo, de modo que la periodicidad responde a un ciclo de $6,11,6$ y 5 años.

${ }^{5}$ Marca creada en el año 1993 por la administración gallega, con la finalidad no solamente de atraer a un turista religioso, sino también a todo tipo de personas que buscasen una nueva experiencia relacionada con la naturaleza, con el deporte o con la cultura en Galicia.
} 
tourism product, drawing particular reference the actions developed during the last Holy Year or Xacobeo 2010.

To carry out this research, the methodology used is based on the use of primary sources, which have been based on the design and conduct in-depth interviews with a group of people linked in one way or another, with the Way of St. James and its communication.

Keywords: Communication, Promotion, Tourism Products, Way of St. James. 


\section{LA IMPORTANCIA DE LA COMUNICACIÓN Y SUS HERRAMIENTAS}

El concepto de Comunicación ha sido desarrollado por multitud de autores. Entre ellos, una de las definiciones más simples es la de Reinares y Calvo (1999) quienes la definen como el paso de una determinada información de unas personas o entidades a otras.

Centrándonos en el caso particular del turismo, es conveniente señalar que la comunicación es una de las herramientas más importantes con las que se cuenta y, en consecuencia, ésta debe de ser planificada y gestionada según unos criterios racionales, que busquen una coherencia absoluta con los planteamientos estratégicos de la empresa o entidad (Iglesias, Talón, García Viana, 2007).

En la actualidad la competitividad existente entre los distintos productos y destinos turísticos hace que no sea suficiente mantener una buena calidad y oferta acerca de los mismos. Es necesario, además, que se dé a conocer la máxima información, con el fin de transmitir sus ventajas y características diferenciadoras, y lograr así que la imagen transmitida sea la deseada y correcta.

Según Rey (2004), los objetivos de la comunicación son:

- Crear conocimiento por medio de la información emitida por la propia empresa u organismo gestor del producto.

- Transmitir las posibilidades que tiene el producto de ofrecer soluciones a necesidades o problemas específicos, al mismo tiempo que trata de crear interés hacia ellos.

- Inducir al futuro cliente a que pruebe o adquiera el producto que se le ofrece.

Este mismo autor define también el proceso de comunicación como el conjunto de señales emitidas por la organización a sus diferentes públicos con el objeto de obtener una respuesta.

El proceso se compone así de los siguientes elementos:

Figura 1.1: El proceso de comunicación 


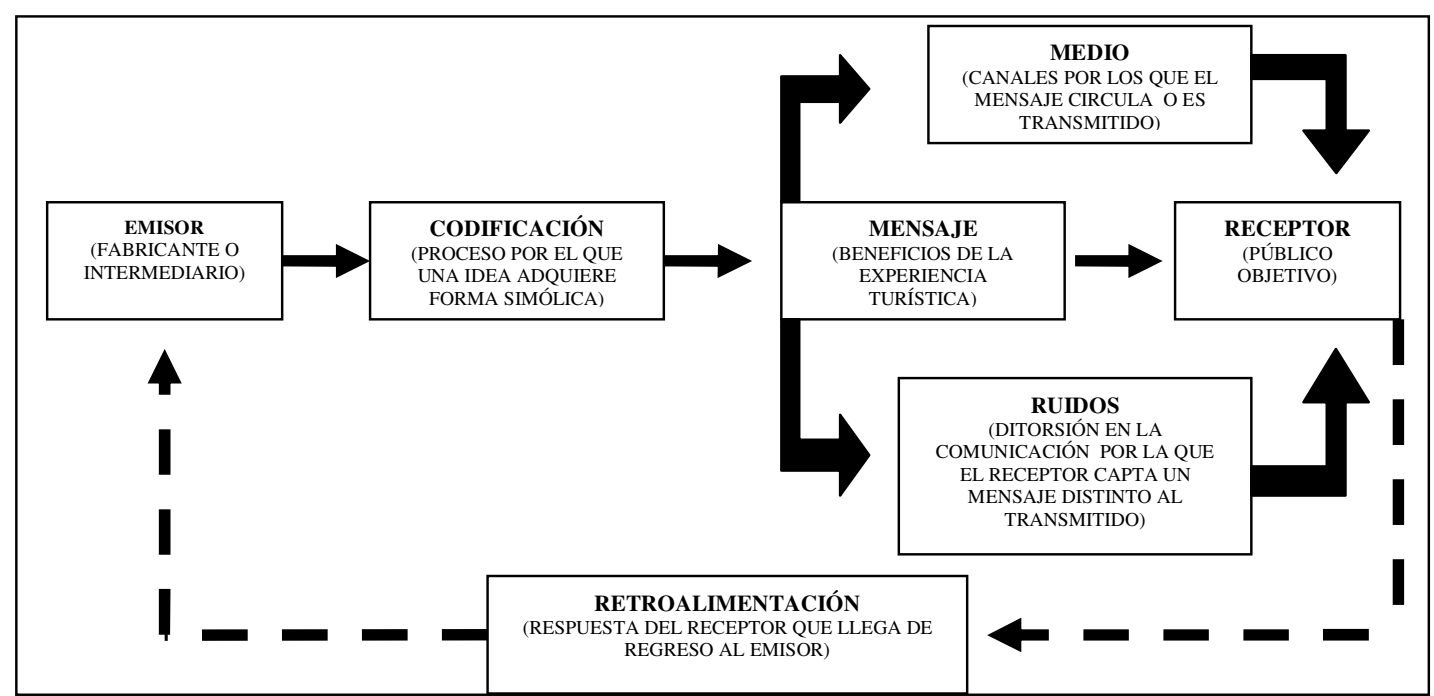

Fuente: Elaboración propia a partir de Rey (2004)

La elección de herramientas de comunicación para promocionar los productos o destinos es un tema que debe ser tratado con sumo cuidado, para evitar caer en desembolsos innecesarios consecuencia de una decisión inadecuada.

INFOADEX ${ }^{7}$ establece así una clasificación de las herramientas de comunicación comercial en lo que respecta a los medios publicitarios, diferenciando por un lado entre los medios convencionales y por otro entre los no convencionales:

Cuadro 1.1.: Clasificación de los medios de comunicación según INFOADEX.

\begin{tabular}{|l|l|}
\hline Medios Convencionales & Medios No convencionales \\
\hline Televisión & Mailing personalizado \\
Dominicales & Buzoneo/folletos \\
Revistas & Marketing telefónico \\
Radio & Regalos publicitarios. \\
Exterior & Publicidad en el punto de venta, rótulos \\
Cine & Ferias y exposiciones \\
Internet & Patrocinio, mecenazgo y marketing social \\
\hline
\end{tabular}

${ }^{7}$ Empresa que se dedica a realizar el control y análisis de la publicidad (ADEX) en España. Se encarga de controlar, archivar y analizar todos los medios convencionales: Cine, Diarios, Exterior, Internet, Radio, Revistas, Dominicales y Televisión.

REDMARKA UIMA-Universidad de A Coruña - CIECID 


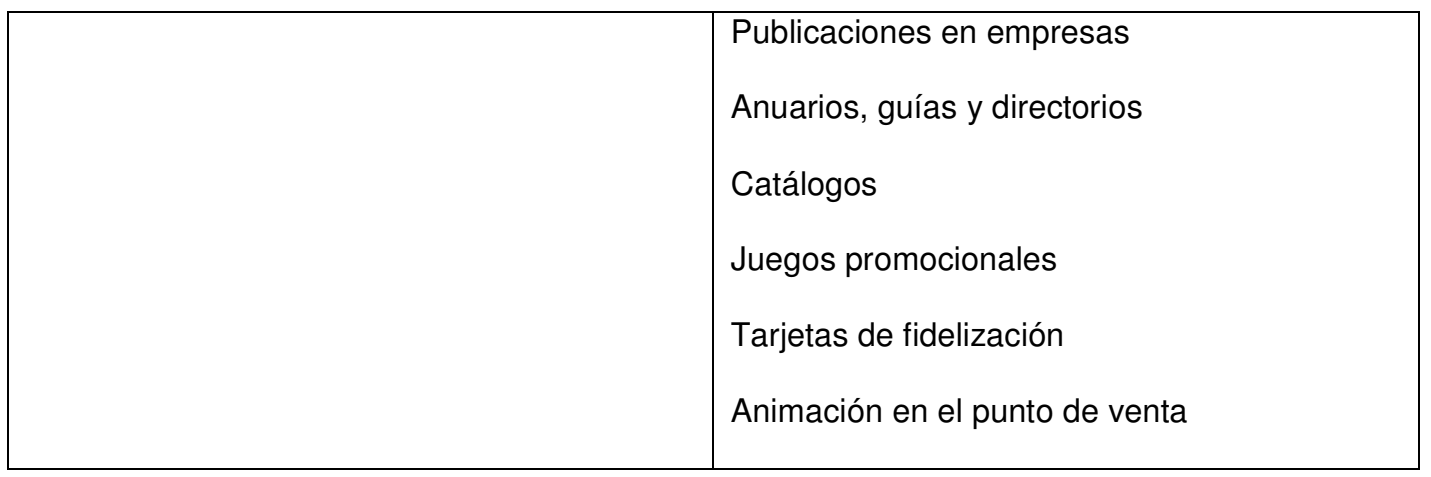

Fuente: Elaboración propia a partir de INFOADEX.

En este sentido la comunicación como variable del marketing mix desde el punto de vista turístico integra principalmente técnicas como la publicidad (convencional, en Internet, folletos y catálogos), la promoción de ventas, las relaciones públicas, el patrocinio, las ferias y la venta personal, que en la práctica han de actuar conjunta y coordinadamente ayudando a construir una identidad propia y proyectando una imagen adecuada del destino y de la oferta cultural (Bigné, Font y Andreu, 2000).

Sin embargo no hay que obviar la importancia que el Boca a Oreja, Internet y las Redes Sociales han ido alcanzando como medios de información, comunicación y promoción.

El Boca a Oreja designado por Gómez (2007) como marketing de viva voz, puede ser definido como aquella comunicación personal de experiencias a otras personas que pertenecen al área de influencia personal. Esta herramienta de comunicación es hoy en día una de las más influyentes en las decisiones de compra por parte de los consumidores.

También el marketing a través de Internet permite el intercambio de información de una manera rápida y sencilla con cualquier parte del mundo, lo cual la convierte en un instrumento tremendamente atractivo para cualquier destino o producto turístico a la hora de llevar a cabo su promoción.

Cerviño (2006) establece que Internet permite la existencia de un mercado virtual abierto las 24 horas del día, los 365 días del año. De hecho, la tendencia actual respecto al crecimiento del empleo de la red establece que si una empresa, un destino o un producto, no están en la misma, directamente no existen (Fraiz, 2009). 
En este sentido, en primer lugar Internet es un medio útil para obtener información sobre las preferencias individuales de los consumidores y desarrollar un marketing personalizado y adaptado al público objetivo al que se pretende llegar. $\mathrm{Y}$ en segundo lugar, permite realizar ajustes rápidos a la evolución del mercado, al poder variar las características de la oferta en tiempo real (Bigné, 2003).

Del mismo modo en la actualidad, y dentro del marketing en Internet, una de las formas de comunicación que mejor está funcionando es lo que se conoce como marketing social. Consiste en la comunicación y el intercambio de experiencias a través de la red, con el fin de influir de forma contundente en la toma de decisiones de otros clientes, así como en los procesos de información y comercialización de los servicios o productos turísticos.

Este tipo de empleo de la red se conoce también como web 2.0 y es un concepto que está sustituyendo progresivamente a las aplicaciones más tradicionales. Los usuarios interactúan a través de Internet mediante el intercambio de videos, fotografías, experiencias y todo tipo de materiales que serán empleados por otros usuarios a la hora de tomar ciertas decisiones y que formarán parte de los contenidos de Internet junto a las páginas web más tradicionales. Es por tanto un sistema muchos más transparente, en donde los clientes son los que tienen un mayor peso a la hora de informar, provocando, de este modo, un mayor esfuerzo por parte de las empresas a la hora de responder a las expectativas de los consumidores. Algunos de los medios que surgen en torno a la web 2.0, caracterizados por su alcance e interactividad, son los blogs, los servicios de alojamiento de vídeos, redes sociales... (Iglesias, Talón y García - Viana, 2007).

\section{OBJETIVOS E HIPÓTESIS DE LA INVESTIGACIÓN}

El objetivo principal de este artículo consiste en analizar la importancia de la comunicación en la promoción del Camino de Santiago como producto turístico, con especial referencia a las acciones desarrolladas durante el pasado Año Santo o Xacobeo 2010. 
La formulación de hipótesis es un elemento fundamental en cualquier proceso de investigación, por eso, a continuación se detallan las hipótesis de trabajo que subyacen al objetivo principal, y que serán confirmadas o en su caso refutadas, a partir del posterior análisis de la investigación empírica realizada.

Hipótesis I: El medio de información más utilizado para planificar la ruta de peregrinación a Santiago es Internet.

Hipótesis II: El Boca a Oreja (Recomendación) es la principal herramienta de comunicación para realizar el Camino.

Hipótesis III: Las Redes Sociales son una buena oportunidad para promocionar el Camino de Santiago.

Hipótesis IV: La información que se proporciona al turista o peregrino acerca del Camino de Santiago es suficiente.

La metodología de este trabajo como ya se ha comentado se asienta en el uso de las fuentes primarias, mediante la realización de entrevistas en profundidad, Bello, Vázquez y Trespalacios (1996) las definen como aquellas en las que el entrevistador interactúa con un individuo y lo alienta para que exprese con entera libertad sus ideas sobre el tema en estudio. Se pretende ir más allá de las respuestas superficiales obtenidas en los cuestionarios de amplia difusión.

Partiendo de lo anteriormente expuesto, el análisis cualitativo de este artículo incluye un total de ocho entrevistas en profundidad, realizadas durante los meses de mayo, junio y julio de 2010.

Para la realización de las entrevistas en profundidad se ha seleccionado a un grupo de personas, que han estado o están relacionadas de una manera directa o indirecta con el Camino de Santiago y la promoción del mismo.

La razón principal en base a la cual se ha seleccionado a los individuos gira en torno a la interesante información que podían aportar a partir de sus vínculos con los siguientes aspectos:

1. La iglesia.

2. La política. 
3. Los medios de Comunicación.

4. Los puntos de información turística

5. La peregrinación.

Además resulta interesante señalar que la práctica totalidad de los entrevistados habían efectuado el Camino o uno de sus tramos, con lo que la visión que podían aportar era todavía mucho más completa y atrayente.

En el siguiente cuadro se establecen los participantes del estudio cualitativo, así como el tipo de vinculación que han mantenido o mantienen con el Camino de Santiago.

Cuadro 2.1: Entrevistados para la investigación cualitativa

\begin{tabular}{|c|c|c|}
\hline ENTREVISTADO & NOMBRE & VINCULACIÓN \\
\hline E.1. & Germán Arias & $\begin{array}{l}\text { Concejal de cultura y medio } \\
\text { rural del ayuntamiento de Sarria. }\end{array}$ \\
\hline E.2. & Tania Hernández & $\begin{array}{l}\text { Colaboradora del periódico } \\
\text { digital noticiasdelcamino.com y } \\
\text { peregrina en diversas } \\
\text { ocasiones. }\end{array}$ \\
\hline E.3. & Cristóbal Ramírez & $\begin{array}{l}\text { Periodista y Presidente de la } \\
\text { Asociación de periodistas y } \\
\text { estudiosos del Camino de } \\
\text { Santiago }\end{array}$ \\
\hline E.4. & Silvia Rozas & $\begin{array}{l}\text { Directora de la secretaría de } \\
\text { medios de comunicación del } \\
\text { Arzobispado de Santiago. }\end{array}$ \\
\hline E.5. & Ignacio Santos & $\begin{array}{l}\text { Anterior gerente de la S. A. de } \\
\text { Gestión del Xacobeo. }\end{array}$ \\
\hline E.6. & $\begin{array}{l}\text { Trabajadora de la oficina de } \\
\text { Turgalicia y Xacobeo (No } \\
\text { ha querido facilitar el }\end{array}$ & $\begin{array}{l}\text { Personal encargado de la } \\
\text { Oficina de Turgalicia y Xacobeo } \\
\text { de la Rúa do Vilar. }\end{array}$ \\
\hline
\end{tabular}




\begin{tabular}{|l|l|l|}
\hline & nombre) & \\
\hline E.7. & Gorona G. & $\begin{array}{l}\text { Peregrina extranjera del Camino } \\
\text { de Santiago procedente de } \\
\text { Toronto. }\end{array}$ \\
\hline E.8. & Lucía Sánchez. & $\begin{array}{l}\text { Peregrina nacional del Camino } \\
\text { de Santiago procedente de } \\
\text { Granada. }\end{array}$ \\
\hline
\end{tabular}

Fuente: Elaboración propia

Cabe precisar que las ocho entrevistas realizadas se han fundamentado en la realización de entrevistas semiestructuradas (Grande y Abascal, 2000; Delgado y Gutiérrez, 1994) sobre la base de un guión común.

\section{RESULTADOS DE LA INVESTIGACIÓN: IMPORTANCIA DE LA COMUNICACIÓN EN LA PROMOCIÓN DEL CAMINO DE SANTIAGO A TRAVÉS DE FUENTES PRIMARIAS}

En el epígrafe anterior y cuadro 2.1., ya se ha explicitado el nombre de las personas entrevistadas, así como la vinculación de los mismos de acuerdo con el tema en cuestión.

De manera que la obtención de información a través de la realización de las entrevistas no era otra que la de conocer las opiniones y actitudes que tenían todas estas personas que de una manera directa o indirecta habían estado 0 estaban en contacto con el Camino de Santiago, la comunicación y promoción.

Tras mostrarle al entrevistado la importancia que representaba el Camino de Santiago para una comunidad autónoma como es Galicia, se procedía a formularle las preguntas.

A continuación se exponen por lo tanto, algunos de los resultados obtenidos a partir de las reuniones llevadas a cabo con cada una de las personas anteriormente citadas. Dichos resultados tienen como finalidad proporcionar 
información suficiente para poder analizar con veracidad el objetivo propuesto y poder posteriormente contrastar las hipótesis formuladas.

\begin{tabular}{|c|c|c|c|c|c|c|c|c|}
\hline \multirow{2}{*}{$\begin{array}{l}\text { Importancia Herramientas de } \\
\text { Comunicación de } 0 \text { a } 5\end{array}$} & \multicolumn{8}{|c|}{ Entrevistados } \\
\hline & E.1 & E.2. & E.3. & E.4. & E.5. & E.6. & E.7. & E.8. \\
\hline Internet / Nuevas Tecnologías & 4 & 5 & 5 & 5 & 5 & 5 & 5 & 5 \\
\hline Boca a Oreja/ Recomendación & 3 & 5 & 4 & 5 & 5 & 5 & 4 & 5 \\
\hline Prescriptores & 2 & 4 & 3 & 4 & 4 & 2 & 4 & 4 \\
\hline $\begin{array}{l}\text { Publicidad en medios de } \\
\text { comunicación }\end{array}$ & 3 & 3 & 2 & 3 & 4 & 5 & 3 & 4 \\
\hline Ferias Tcas./Expo./Congresos & 2 & 3 & 2 & 4 & 4 & 4 & 4 & 3 \\
\hline RRPP/Viajes de familiarización & 2 & 1 & 1 & 4 & 4 & 2 & 4 & 3 \\
\hline $\begin{array}{l}\text { Promociones de ventas/ } \\
\text { Merchandising }\end{array}$ & 2 & 2 & 0 & 3 & 3 & 1 & 1 & 2 \\
\hline Esponsorización o Patrocinio & 2 & 3 & 1 & 2 & 3 & 5 & 0 & 3 \\
\hline $\begin{array}{l}\text { Folleto turístico y otros } \\
\text { materiales impresos }\end{array}$ & 3 & 4 & 4 & 4 & 4 & 5 & 5 & 3 \\
\hline
\end{tabular}

Cuadro 3.1: Resultados de las entrevistas en profundidad

Fuente: Elaboración propia 
Tras preguntarle a los entrevistados que valorasen la importancia de las herramientas de comunicación utilizadas para comunicar y promocionar el Camino de Santiago, en una escala de 0 a $5^{8}$. Los resultados obtenidos ponen de manifiesto que Internet y las nuevas tecnologías son las herramientas de comunicación más relevantes. Tras ellas, el boca a oreja / recomendación, y los folletos son también muy importantes.

Por el contrario, el patrocinio y las promociones de venta, son considerados por los entrevistados, como las herramientas menos eficaces a la hora de promocionar y atraer al público objetivo hacia el Camino.

\footnotetext{
${ }^{8}$ Representando 0 ninguna importancia y 5 una gran importancia.
} 
Cuadro 3.2: Resultados de las entrevistas en profundidad

Fuente: Elaboración propia

En base a las opiniones aportadas por parte de cada uno de los entrevistados,

\begin{tabular}{|c|c|}
\hline Entrevistado & $\begin{array}{l}\text { ¿Llegará Internet a solapar la utilización de otras herramientas más } \\
\text { tradicionales en la comunicación del Camino? }\end{array}$ \\
\hline E.1. & "No, puede complementarlas, pero nunca sustituirlas." \\
\hline E.2. & $\begin{array}{l}\text { "No, será algo complementario, pero en mi opinión no llegará a } \\
\text { solaparlas." }\end{array}$ \\
\hline E.3. & $\begin{array}{l}\text { "Creo que no, son complementarios y cada uno cumple una función } \\
\text { diferente." }\end{array}$ \\
\hline E.4. & $\begin{array}{l}\text { "Sí, sin lugar a dudas. Y de hecho lo hace, ya que hoy en día hasta el } \\
\text { folleto se cuelga en Internet." }\end{array}$ \\
\hline E.5. & $\begin{array}{l}\text { "Existen diversos targets que no dominan tanto el uso de Internet y, por } \\
\text { tanto, son necesarias herramientas más tradicionales para acceder a } \\
\text { ellos." }\end{array}$ \\
\hline E.6. & $\begin{array}{l}\text { "Son complementarios a la hora de planificar el Camino, con lo cual no } \\
\text { deberían de solaparse, sino complementarse entre sí." }\end{array}$ \\
\hline E.7. & $\begin{array}{l}\text { "Creo que Internet en un futuro será insustituible, pero también pienso } \\
\text { que las herramientas como los folletos nunca pasarán de moda y } \\
\text { siempre serán útiles." }\end{array}$ \\
\hline E.8. & $\begin{array}{l}\text { "Creo que no, en tal caso será complementaria la utilización de unos y } \\
\text { otras." }\end{array}$ \\
\hline \multicolumn{2}{|c|}{$\begin{array}{l}\text { en relación a la posibilidad de que Internet pueda solapar a las herramientas de } \\
\text { comunicación tradicionales, la mayoría de ellos consideran que aunque el usc } \\
\text { de Internet es y será insustituible, no llegará a hacer desaparecer a las } \\
\text { herramientas de comunicación más tradicionales (folletos, publicidad en medios } \\
\text { de comunicación...). }\end{array}$} \\
\hline
\end{tabular}


En este sentido, excepto para uno de los entrevistados (E.4.), que destaca que Internet sí que se convertirá en la herramienta clave a la hora de planificar la comunicación de un producto o destino; todos los demás justifican la importancia de las otras técnicas de comunicación, al mismo tiempo que aclaran que su utilización y la de Internet son claramente complementarios y que nunca tienen porque llegar a solaparse. 
Cuadro 3.3: Resultados de las entrevistas en profundidad

\begin{tabular}{|c|c|}
\hline Entrevistado & Actuaciones para lograr que el Camino sea más conocido. \\
\hline E.1. & $\begin{array}{l}\text { "Se debe especial hincapié en la mejora de la promoción del Camino, tratando } \\
\text { de que llegue al mayor número de personas posibles, de forma adecuada." }\end{array}$ \\
\hline E.2. & $\begin{array}{c}\text { "A nivel nacional, la gente se queja de no recibir mucha más información del } \\
\text { Camino. A nivel internacional, la información les llega en base a ferias } \\
\text { especializadas de turismo y también por los prescriptores." }\end{array}$ \\
\hline E.3. & $\begin{array}{l}\text { "Lo primero es cuidar el Camino. También hay que promocionarlo allá donde } \\
\text { hay asociaciones de amigos del Camino de Santiago y, finalmente, empleando } \\
\text { los fondos públicos para las promociones constantes en el exterior." }\end{array}$ \\
\hline E.4. & $\begin{array}{l}\text { "Yo apuesto mucho por el tema de las películas; el cine mueve a mucha gente. } \\
\text { Pero siempre es peligroso porque la imagen ofrecida puede no ser buena. } \\
\text { También creo que la promoción debería hacer bastante hincapié en aquellos } \\
\text { destinos en donde el Camino no es tan relevante." }\end{array}$ \\
\hline E.5. & $\begin{array}{l}\text { "La clave es no dejar de trabajar la imagen del Camino, independientemente de } \\
\text { que sea Año Santo o no. Con un trabajo constante de promoción y puesta a } \\
\text { punto, el Camino se afianzará y se expandirá constantemente, tanto a nivel } \\
\text { nacional como internacional." }\end{array}$ \\
\hline E.6. & $\begin{array}{l}\text { "Creo que se debería trabajar específicamente con ciertos sectores de turistas } \\
\text { que tal vez estén un poco más descuidados como por ejemplo los niños. } \\
\text { Además, la recuperación de trazados con fuerte tradición histórica, sería otra } \\
\text { medida interesante." }\end{array}$ \\
\hline E.7. & $\begin{array}{c}\text { "Creo que se debería distribuir más información en las oficinas turísticas de } \\
\text { todos los países. También, considero que hoy en día, a través de las nuevas } \\
\text { tecnologías se puede desarrollar una promoción de sensaciones o experiencia } \\
\text { que realmente transfiera parte de la esencia del Camino." }\end{array}$ \\
\hline E.8. & $\begin{array}{c}\text { "Creo que antes de pensar en promocionar, se debería crear un buen producto. } \\
\text { En definitiva, la primera actuación debe de ser la mejora del Camino; luego, la } \\
\text { promoción viene sola." }\end{array}$ \\
\hline
\end{tabular}

Fuente: Elaboración propia

REDMARKA UIMA-Universidad de A Coruña - CIECID 
Han sido varias las acciones propuestas por parte de los entrevistados para mejorar la comunicación del Camino con el fin de que sea todavía más conocido y alcance un buen posicionamiento tanto a nivel nacional como internacional.

Así por un lado, (E.3. y E.8.), establecen que a lo que primero a lo que se le debería prestar especial atención es a la protección y cuidado del Camino de modo que le permita convertirse en un producto bien valorado y preparado, y ser así capaz de atraer a multitud de personas, sin necesidad de ningún otro tipo de acción comunicativa.

Por otro lado (E.2. y E.7.) consideran que la difusión de información más completa y que llegue a más lugares, es tremendamente necesaria si lo que se pretende es que el producto alcance una considerable proyección mundial. Además, también resaltan el valor de desarrollar una política de promoción constante a lo largo del tiempo y coherente con los lugares en los que se vaya a implantar.

Finalmente, otros de los entrevistados proponen actuaciones más concretas como la apuesta por los prescriptores y las ferias turísticas como motores de atracción de público internacional (E.2.; E.4.); el trabajar con ciertos sectores dentro del público objetivo que tal vez están siendo olvidados y a los que se les debería de prestar especial atención (E. 6.); o bien el desarrollo de actividades que ofrezcan la posibilidad de descubrir la esencia del Camino a través de los sentidos: el tacto, el olfato, la vista... siendo todo ello posible gracias a las nuevas tecnologías y técnicas de la información (E.7.). 
Cuadro 3.4: Resultados de las entrevistas en profundidad

\begin{tabular}{|c|c|}
\hline Entrevistado & $\begin{array}{l}\text { Promoción de las otras rutas de peregrinación }{ }^{9} \text { existentes en el } \\
\text { Camino. }\end{array}$ \\
\hline E.1. & $\begin{array}{c}\text { "Creo que las rutas tradicionales son las que tienen su razón de ser ligadas } \\
\text { al Camino; sin embargo, el resto, considero que son sólo inventos } \\
\text { puramente turísticos." }\end{array}$ \\
\hline E.2. & $\begin{array}{l}\text { "Creo que se deberían de promocionar todas las rutas por igual. De hecho, } \\
\text { las más tradicionales ya cuentan con el 'apoyo' de los peregrinos que las } \\
\text { han recorrido. Otras vías, no tan conocidas, necesitan del impulso que fue } \\
\text { dado al Camino francés con anterioridad." }\end{array}$ \\
\hline E.3. & $\begin{array}{l}\text { “La promoción se debe de concentrar en uno o en unos pocos, y } \\
\text { especializar el resto. Además, es imposible hablar de promoción de todas } \\
\text { las rutas, puesto que ni se tiene el dinero suficiente para hacerlo, ni se } \\
\text { lograría obtener la comprensión del lector." }\end{array}$ \\
\hline E.4. & $\begin{array}{c}\text { "Yo promocionaría las rutas que no sea la francesa, puesto que está } \\
\text { saturada." }\end{array}$ \\
\hline E.5. & $\begin{array}{c}\text { "Todas las rutas de peregrinación merecen una especial atención y trato. Se } \\
\text { deben de dar a conocer todas ellas para tratar de descongestionar las más } \\
\text { importantes." }\end{array}$ \\
\hline E.6. & $\begin{array}{l}\text { "Considero que la cuestión en base a estas nuevas rutas se centra } \\
\text { fundamentalmente en dos ejes: la necesidad urgente de descongestionar el } \\
\text { Camino francés y la búsqueda de nuevas experiencias que se puedan } \\
\text { ofrecer a los peregrinos que ya hayan hecho un Camino." }\end{array}$ \\
\hline E.7. & $\begin{array}{l}\text { "Creo que todas son importantes, y por tanto que todas merecen una } \\
\text { especial atención." }\end{array}$ \\
\hline E.8. & $\begin{array}{l}\text { "Creo que se deben de promocionar todas, es más, incluso creo que se les } \\
\text { debe conceder especial importancia a las nuevas que han ido apareciendo." }\end{array}$ \\
\hline
\end{tabular}

${ }^{9}$ Estas rutas a las que nos referimos son las siguientes: el Camino Inglés, el Camino Portugués, el Camino del Norte, el Camino Primitivo, el Camino del Sudeste o Vía de la Plata, el Camino de Fisterra y la Ruta del Mar de Arousa y Río Ulla. 
En lo relativo a la comunicación de las nuevas rutas de peregrinación que han ido surgiendo con el paso de los años, se pone de manifiesto que la mayor parte de los entrevistados (E.2., E.4., E.5., E.6., E.7., E.8. y E.9.) consideran relevante su promoción, sobre todo por dos motivos básicos: el descongestionar los caminos más tradicionales, como el Camino Francés, y el ofrecer alternativas al peregrino que quiera volver a hacer el Camino sin repetir el mismo itinerario.

Dos de los entrevistados discrepan del resto en este aspecto alegando fundamentalmente dos motivos: por un lado (E.1), que la promoción del Camino debería centrarse en las rutas más tradicionales, puesto que el resto no son más que recursos turísticos alejados de la esencia del verdadero Camino; y por otro lado (E.3.), que se debería promocionar uno o unos pocos Caminos, tratando de dar un uso específico al resto. Además este último entrevistado (E.3.), también resalta la imposibilidad de promocionar todas las rutas por igual, ya que primeramente no hay suficiente dinero y en segundo lugar no se logaría obtener la comprensión del propio público.

A la vista de los resultados se puede precisar hasta el momento la importancia que Internet representa para los entrevistados como medio de información y promoción, así como la necesidad de llevar a cabo acciones concretas de planificación y comunicación en el Camino de Santiago para dar a conocer esta ruta de peregrinación milenaria que tantos beneficios reporta para Galicia desde diferentes perspectivas.

A continuación se procede a la contrastación de las hipótesis formuladas previamente con el fin de obtener unos resultados más precisos en relación al objetivo del presente artículo. Las siguientes preguntas de la entrevista permiten corroborar o en su caso refutar las hipótesis establecidas en este artículo que a continuación se detallan. 


\section{Hipótesis I: El medio de información más utilizado para planificar la ruta de peregrinación a Santiago es Internet.}

Cuadro 3.5: Resultados de las entrevistas en profundidad

\begin{tabular}{|c|c|}
\hline Entrevistado & $\begin{array}{l}\text { Medio de información más empleado por los peregrinos para la } \\
\text { planificación del viaje. }\end{array}$ \\
\hline E.1. & "Internet y las guías publicadas al respecto." \\
\hline E.2. & $\begin{array}{c}\text { "Internet, destacando la página de Eroski-Consumer, así como los } \\
\text { foros y guías turísticas en soporte papel." }\end{array}$ \\
\hline E.3. & "Internet, sin ninguna duda." \\
\hline E.4. & "Internet, pero también las agencias de viaje." \\
\hline E.5. & "Internet es una de las herramientas que más sobresale." \\
\hline E.6. & "Internet, las guías especializadas y los folletos." \\
\hline E.7. & "Creo que Internet, desde luego en mi caso fue así." \\
\hline E.8. & "Internet, sin ninguna duda." \\
\hline
\end{tabular}

Fuente: Elaboración propia

A partir de los resultados obtenidos se puede afirmar que Internet se posiciona, claramente, como el principal medio de información a la hora de planificar el Camino de Santiago. Todos los entrevistados han coincidido en resaltar la importancia que en la actualidad representa esta herramienta a la hora de informarse y planificar el itinerario.

También enumeran como segunda opción ciertas herramientas como las guías, los folletos o bien las propias consultas en agencias de viaje, pero aun así no cabe duda de que la Hipótesis I se confirma. 


\section{Hipótesis II: El Boca a Oreja/ recomendación es la principal herramienta de comunicación para realizar el Camino.}

Cuadro 3.6: Resultados de las entrevistas en profundidad

\begin{tabular}{|c|c|}
\hline $\begin{array}{c}\text { Entrevistado } \\
\text { E.1. }\end{array}$ & $\begin{array}{r}\text { Boca a oreja, prescriptores, Internet y folletos. ¿Cuál es la más } \\
\text { importante en la promoción del Camino? }\end{array}$ \\
\hline E.2. & "Internet y folletos. No podría hacer ninguna distinción entre ellos." \\
\hline E.3. & "Internet y, en segundo lugar los folletos." \\
\hline E.4. & "Sin lugar a dudas, Internet, complementado por el boca a oreja y \\
\hline E.5. & "Internet, sin lugar a dudas." \\
\hline E.6. & "Sin lugar a dudas, Internet." \\
\hline E.7. & \\
\hline
\end{tabular}

Fuente: Elaboración propia

A la vista de los resultados el Boca a Oreja o recomendación no es considerado como la principal herramienta de comunicación a la hora de promocionar el Camino de Santiago, puesto que la mayoría de los entrevistados han coincidido en destacar que es Internet quien se vuelve a posicionar en primer lugar por encima del resto de herramientas, poniendo así de manifiesto, su importancia y dominio en torno a la comunicación actual. No confirmándose por tanto en este caso la Hipótesis II. 


\section{Hipótesis III: Las redes sociales son una buena oportunidad para promocionar el Camino de Santiago.}

Cuadro 3.7: Resultados de las entrevistas en profundidad

\begin{tabular}{|c|c|}
\hline Entrevistado & $\begin{array}{l}\text { Las redes sociales como oportunidad para dar a conocer o } \\
\qquad \text { promocionar el Camino. }\end{array}$ \\
\hline E.1. & $\begin{array}{l}\text { "Por supuesto, estar en las redes sociales es tan básico como lo fue en su } \\
\text { momento tener una página web." }\end{array}$ \\
\hline E.2. & $\begin{array}{l}\text { "Siempre son buenas oportunidades. Es una buena forma para acercarse a } \\
\text { gente que de otra forma sería imposible alcanzar." }\end{array}$ \\
\hline E.3. & $\begin{array}{l}\text { "Hay que estar en las redes sociales, porque es peor no estar, pero no porque } \\
\text { esto vaya a atraer a nadie a hacer el Camino." }\end{array}$ \\
\hline E.4. & "Sí, pero sólo entre la gente joven." \\
\hline E.5. & $\begin{array}{c}\text { "En la actualidad es fundamental trabajar con las redes sociales. Permiten llegar } \\
\text { a todo el mundo con una inversión muy reducida y prácticamente en el momento } \\
\text { en que se quiera." }\end{array}$ \\
\hline E.6. & $\begin{array}{l}\text { "Sí, ya que hoy en día cada vez son más las personas que visitan este tipo de } \\
\text { redes y, por tanto, se puede llegar a prácticamente todo el mundo." }\end{array}$ \\
\hline E.7. & $\begin{array}{c}\text { "Realmente creo que es importante e interesante, porque se puede llegar a } \\
\text { millones de personas con mucha facilidad." }\end{array}$ \\
\hline E.8. & $\begin{array}{c}\text { "Personalmente, creo que no. Al introducir el Camino en las redes sociales, se } \\
\text { está masificando y pierde totalmente su esencia." }\end{array}$ \\
\hline
\end{tabular}

Fuente: Elaboración propia

Los resultados que ofrecen las entrevistas en profundidad confirman la Hipótesis III, de tal manera que las redes sociales son consideradas una buena oportunidad para lograr acceder a unos targets concretos que a través de otros medios sería complicado alcanzar con tanta facilidad y en un periodo 
de tiempo tan reducido, además, de la ventaja que representa en cuanto a la inversión, en comparación con otras herramientas, lo que las hace adecuadas para promocionar el Camino.

\section{Hipótesis IV: La información que se proporciona al turista o peregrino acerca del Camino de Santiago es suficiente.}

\section{Cuadro 3.8: Resultados de las entrevistas}

\begin{tabular}{|c|c|}
\hline Entrevistado & La información que se ofrece del Camino ¿es la suficiente? \\
\hline E.1. & $\begin{array}{c}\text { "Obviamente, todo es mejorable y la información no es una excepción, creo que ésta } \\
\text { debería de mejorarse y ampliarse" }\end{array}$ \\
\hline E.2. & "Es suficiente, aunque deberían existir más canales de información." \\
\hline E.3. & $\begin{array}{l}\text { "Debe diferenciarse entre información pura y dura, con datos objetivos, y los } \\
\text { testimonios subjetivos, que en la mayoría de los casos no son más que ruido. } \\
\text { Quienes deberían dar buena información (los organismos oficiales) no la dan. Y las } \\
\text { Asociaciones de Amigos del Camino no tienen webs que faciliten la información." }\end{array}$ \\
\hline E.4. & "Se debería mejorar, y ampliar considerablemente." \\
\hline E.5. & $\begin{array}{c}\text { "La información que se le ofrece al peregrino antes de iniciar su viaje es suficiente, } \\
\text { sin embargo, una vez que inician el Camino, es mucho más complicado llegar a ellos, } \\
\text { pero estamos trabajando por hacerlo bien." }\end{array}$ \\
\hline E.6. & $\begin{array}{l}\text { "La información que se ofrece debe de estar actualizada constantemente y considero } \\
\text { que esto se está logrando con la del Camino, por lo que considero que es suficiente." }\end{array}$ \\
\hline E.7. & "Considero que es buena y suficiente, sin problemas." \\
\hline E.8. & $\begin{array}{c}\text { "Creo que todo es mejorable, pero creo que sería interesante que se facilitase más } \\
\text { información para llegar hasta Galicia y, sobre todo, para llegar a los centros de salud } \\
\text { y hospitales." }\end{array}$ \\
\hline
\end{tabular}

Fuente: Elaboración propia 
En base a los resultados que desprende la investigación cualitativa no se considera suficiente la información que se proporciona al turista o peregrino acerca del Camino de Santiago. Es importante por lo tanto, cuidar ciertos aspectos relativos a la información si se desea que el Camino siga generando para Galicia los beneficios que hoy reporta. Cabe resaltar que, pese a todo, ésta fue la hipótesis de mayor controversia puesto que la mitad de los entrevistados consideraron oportuna su aceptación y la otra mitad la refutaron. Se refuta por tanto esta Hipótesis IV.

\section{CONCLUSIONES}

El Camino de Santiago se ha convertido en la actualidad en el producto turístico estrella de Galicia, capaz de atraer a millones de personas por motivos tan diversos como la religión o el puro ocio.

Una correcta y veraz comunicación del Camino es uno de los factores esenciales en cuanto a su imagen y posicionamiento. A pesar de ello, hay otros aspectos básicos que inciden de forma irremediable en ambos, tal y como es el caso del cuidado y protección de los caminos, puesto que si no hay un buen recurso no hay un buen producto. Además la imagen del Camino ha de trabajarse desde una política común y constante a lo largo del tiempo, de tal forma que logre asentar unos principios básicos, al mismo tiempo que marque el posicionamiento adecuado que debería ostentar el Camino.

Por un lado, a la hora de comunicar y promocionar esta ruta de peregrinación, Internet y las nuevas tecnologías son las herramientas clave a tener en cuenta, y a las que en consecuencia, más atención se han de prestar puesto que en ciertos momentos y para determinados aspectos la información que se proporciona a través de los medios no es ni la suficiente, ni la adecuada. A pesar de ello tampoco se puede olvidar que elementos tan tradicionales como los folletos o las guías son imprescindibles como complemento comunicativo. 
Por otro lado, la comunicación de las diversas rutas Jacobeas ${ }^{10}$ que han ido surgiendo en la actualidad tiene como fin último la descongestión de los Caminos más demandados y el dar a conocer a todos los turistas o peregrinos que viajan a Compostela nuevas rutas alternativas a las principales vías, por lo que también es interesante e importante apostar por ellas.

En definitiva, debe ser esencial planificar y difundir una correcta y adecuada comunicación acerca del Camino de Santiago para que el producto siga gozando en un futuro del éxito que ostenta en la actualidad.

\section{BIBLIOGRAFÍA}

AGUIRRE, M.S. (Coord.) (2000): Marketing en sectores específicos. Pirámide. Madrid.

BELLO, L.; VÁZQUEZ, R. y TRESPALACIOS, J.A. (1996): Investigación de mercados y estrategia de marketing. Civitas. Madrid.

BIGNÉ, E., FONT, X., y ANDREU, L. (2000): Marketing de destinos turísticos. Análisis y estrategias de desarrollo. ESIC. Madrid.

BIGNÉ, E. (2003): Promoción comercial, ESIC, Madrid.

CERVIÑO, J. (2006): Marketing internacional: Nuevas perspectivas para un mercado globalizado, Pirámide, España.

DELGADO, J.M., y GUTIÉRREZ, J. (1994): Metodología de las ciencias del comportamiento: Métodos y técnicas cualitativas de investigación en ciencias sociales. Síntesis Psicología. Madrid.

FRAIZ BREA, J. A. (2009): "Nuevas estrategias de comunicación para la generación y proyección de la imagen de destinos turísticos." Universidad de Vigo, en XIV Congreso AECIT Retos para el Turismo español. Cambio de paradigma. Gijón, 18, 19 y 20 de noviembre de 2009.

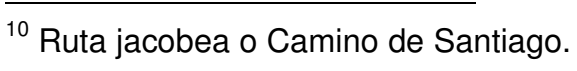


GÓMEZ, A. (2007): "Análisis de los activos del valor de marca turística: diferenciación, gestión de imagen, calidad percibida, fidelización, el marketing de viva voz y la comunicación integrada". Anuario Jurídico y Económico Escurialense, pp. 591-630.

GRANDE, I., y ABASCAL, E. (2000): Fundamentos y técnicas de investigación comercial. 5aㅗ edición. ESIC. Madrid.

IGLESIAS, TALÓN Y GARCÍA (2007) Comercialización de productos y servicios turísticos. Síntesis. Madrid.

MARCHENA, M. (1993): "El Camino de Santiago como producto turístico: comunidades autónomas y promoción del turismo". Los caminos de Santiago y el territorio. Santiago, pp. 909-936.

REINARES LARA, P. y CALVO FERNÁNDEZ. S. (1999): Gestión de la comunicación comercial. McGraw-Hill. Madrid.

REY, M., et al. (2004): Fundamentos de Marketing Turístico. Síntesis. Madrid.

\section{WEBS UTILIZADAS}

http://caminodesantiago.consumer.es

http://www.santiagoturismo.com

http://www.turgalicia.es

http://www.peregrinossantiago.es

http://www.xacobeo.es

http://www.noticiascamino.com

Para citar este artículo:

Mondelo López, Silvia - Rodríguez Fernández, Magdalena (29-07-2011). ANÁLISIS DE LA COMUNICACIÓN EN LA PROMOCIÓN DEL CAMINO DE SANTIAGO COMO PRODUCTO TURÍSTICO.

REDMARKA - CIECID - Unidad de Investigación en Marketing Aplicado- Universidad de A Coruña

Año III, Número 6, V1, pp.59-83

ISSN 1852-2300

URL del Documento: cienciared.com.ar/ra/doc.php?n=1491

URL de la Revista: cienciared.com.ar/ra/revista.php?wid=39 